\title{
5'-Adenosine Monophosphate Is the Neutrophil-derived Paracrine Factor that Elicits Chloride Secretion from T84 Intestinal Epithelial Cell Monolayers
}

\author{
James L. Madara, * Thomas W. Patapoff, ${ }^{*}$ Beth Gillece-Castro, ${ }^{\star}$ Sean P. Colgan, ${ }^{*}$ \\ Charles A. Parkos, * Charlene Delp, * and Randall J. Mrsny \\ * Division of Gastrointestinal Pathology, Department of Pathology, Brigham and Women's Hospital, and Harvard Medical School, and \\ the Harvard Digestive Diseases Center, Boston, Massachusetts 02115; and ${ }^{\ddagger}$ Genentech, Inc., South San Francisco, California 94080
}

\begin{abstract}
Neutrophil transmigration across intestinal epithelia is thought to contribute to epithelial dysfunction and characterizes many inflammatory intestinal diseases. Neutrophils activated by factors, normally present in the lumen, release a neutrophilderived secretagogue activity to which intestinal epithelia respond with an electrogenic chloride secretion, the transport event which underlies secretory diarrhea. Using sequential ultrafiltration, column chromatographic, and mass and Raman spectroscopic techniques, neutrophil-derived secretagogue was identified as 5'-AMP. Additional studies suggested that neutrophil-derived 5'-AMP is subsequently converted to adenosine at the epithelial cell surface by ecto-5'-nucleotidase and that adenosine subsequently activates intestinal secretion through adenosine receptors on the apical membrane of target intestinal epithelial cells. These findings suggest that this ATP metabolite may serve as a neutrophil-derived paracrine mediator that contributes to secretory diarrhea in states of intestinal inflammation. (J. Clin. Invest. 1993.91:2320-2325.) Key words: adenosine • electrolyte transport • leukocyte • inflammation
\end{abstract}

\section{Introduction}

Hydration of mucosal surfaces of the mammalian airway, pancreatic tree, and intestinal tract occurs by the transport event of electrogenic chloride secretion (1). In the intestine, the anatomic subsite at which this secretory process occurs is the epithelial lining of the intestinal crypt (2). The crypt is also the site where neutrophils migrate across the epithelium in acute inflammatory states and subsequently cluster in the crypt lumen to form the classic feature of intestinal inflammation, the crypt abscess (3). Crypt abcesses may be modeled by layering isolated neutrophils onto monolayers of a human-derived cryptlike intestinal epithelial cell line, T84 (4-7). Exposure of the apical surface of $\mathrm{T} 84$ monolayers to neutrophils previously activated by agonists endogenous to the crypt lumen, such as

Address correspondence to Dr. James L. Madara, Department of Pathology, Brigham and Women's Hospital, 75 Francis Street, Boston, MA 02115.

Received for publication 8 December 1992.

J. Clin. Invest.

(c) The American Society for Clinical Investigation, Inc.

0021-9738/93/05/2320/06 \$2.00

Volume 91, May 1993, 2320-2325 endotoxin and $\mathrm{n}$-formulated peptides, elicits a short circuit current $\left(I_{s c}\right)^{1}$ due to activation of electrogenic chloride secretion (5-7). This chloride secretory process has the net effect of producing isotonic fluid secretion (1) and likely serves as a primitive defensive response by which mucosal surfaces are "flushed."

Stimulation of chloride secretion by neutrophils does not appear to require physical contact with the intestinal epithelium since buffer conditioned with activated neutrophils can elicit chloride secretion when applied to T84 monolayers (5). This neutrophil-derived secretagogue (NDS) activity is effective predominately at the apical membrane, directly elicits chloride secretion from primary isolates of mammalian intestinal crypt epithelial cells (6), and thus may contribute to the secretory diarrhea that occurs in states characterized by neutrophil migration across the crypt lumen. NDS activity can also be obtained from the human promyelocytic cell line HL-60 and the level of this activity is enhanced when these cells differentiate toward the neutrophil phenotype (7). Like neutrophilderived NDS, that obtained from HL-60 cells is water soluble and can pass through a $1-\mathrm{kD}$ nominal mol wt cutoff filter (7). We now report the purification and identification of NDS. Although several compounds known to be produced by neutrophils can elicit chloride secretion when applied to the target epithelia in pure form at concentrations in the micromolar to millimolar range (8-10), the NDS activity derived from neutrophils and HL-60 cells represents a metabolite not previously recognized as a paracrine mediator which may play a crucial role in neutrophil-intestinal epithelial crosstalk.

\section{Methods}

HL-60 cells were grown in RPMI medium with $10 \%$ ( vol/vol) heat-inactivated fetal calf serum with $2 \mathrm{mM} \mathrm{L-glutamine} \mathrm{(Gibco} \mathrm{Laboratories,}$ Grand Island, NY) in $75-\mathrm{cm}$ tissue culture flasks at $37^{\circ} \mathrm{C}$ and at $5 \%$ $\mathrm{CO}_{2}$. To prepare an enriched source of NDS, cells were washed in HBSS, pelleted at $100 \mathrm{~g}$ for $5 \mathrm{~min}$, and resuspended in distilled water at $10^{8}$ cells $/ \mathrm{ml}$. Clarified lysate supernatant $(12,000 \mathrm{~g}$ for $15 \mathrm{~min})$ was passed through a $1,000 \mathrm{~mol}$ wt cutoff ultrafiltration membrane (Amicon) by 25 psi nitrogen gas.

Human peripheral blood neutrophils were isolated as previously described $(5,6,11)$. A crude NDS-conditioned cell-free supernatant was obtained after neutrophil activation with $0.1 \mu \mathrm{g} / \mathrm{ml}$ phorbol myristate acetate (PMA), exactly as described before (6). This supernatant was also size-filtered as outlined above for the HL-60-derived enriched NDS preparation.

1. Abbreviations used in this paper: $\mathrm{I}_{\mathrm{sc}}$, short circuit current; NDS, neutrophil-derived secretagogue; RP, reverse phase. 
Crude NDS so prepared from HL-60 cells or freshly isolated and activated human neutrophils was then fractionated by a technique we empirically identified as useful in isolating and purifying NDS bioactivity. Briefly, HPLC anion exchange chromatography was performed using a $30 \mathrm{~mm} \times 2.1 \mathrm{~mm}$ DEAE $10-\mu \mathrm{m}$ particle size column (Polypore; Brownlee Labs, Santa Clara, CA). Elution was with either a linear ammonium acetate gradient (100-200 mM in Fig. 1) or a linear (0200 in Fig. 2) phosphate buffer ( $\mathrm{pH} 7.0)$ gradient. After injection $(0.25$ $\mathrm{ml}$ ) the column was washed for $5 \mathrm{~min}$ with either $100 \mathrm{mM}$ ammonium acetate or $1 \mathrm{mM}$ phosphate buffer, then the NDS was eluted over 5 $\min$. Flow rate was $0.5 \mathrm{ml} / \mathrm{min}$. In addition, a reverse phase (RP) HPLC method was used where indicated to quantitate adenosine. Briefly, the RP-HPLC system for adenosine, detected at $254 \mathrm{~nm}$, used a $30 \mathrm{~mm} \times 4.6 \mathrm{~mm}$ OD-300 column (Aquapore; Brownlee Labs). After sample injection, the column was washed with $97.5 \% 50 \mathrm{mM}$ ammonium phosphate at $\mathrm{pH} 4.0$ ( solvent $\mathrm{A}$ ) and $2.5 \%$ methanol (solvent $\mathrm{B}$ ) at $0.25 \mathrm{ml} / \mathrm{min}$ for $6 \mathrm{~min}$. Adenosine was eluted using a linear gradient of $80 \%$ solvent $\mathrm{A} / 20 \%$ solvent $\mathrm{B}$ over $9 \mathrm{~min}$ at $0.5 \mathrm{ml} / \mathrm{min}$. Using this system, 5'-AMP eluted in the void volume and was quantitated by incubating an aliquot of each NDS sample with 5'-nucleotidase (300 $\mathrm{mg}$ ) for $30 \mathrm{~min}$ at $25^{\circ} \mathrm{C}$ before HPLC injection.

T84 cell monolayers were used as previously described $(6,7,11)$ to assess NDS activity of the fractions generated by the above methods. T84 monolayers were plated on collagen-coated $0.33-\mathrm{cm}^{2}$ nucleopore filters, interfaced with apical and basolateral $\mathrm{Ag} / \mathrm{AgCl}$ and Calomel electrodes via agar bridges, and $I_{\mathbf{s c}}$ was determined via the voltage clamp technique as previously described $(6,7,11)$. Fractions obtained from column chromatography were individually lyophylized, reconstituted in HBSS, and the $\mathrm{pH}$ was adjusted if needed to 7.4. Each fraction was reconstituted to a volume equal to the original sample applied to the column and further dilutions made with the above buffer as specifically indicated.

Purified fractions obtained from the DEAE chromatography of the crude NDS were pooled and characterized by mass spectroscopy and other techniques as indicated below. Mass spectra were recorded on a PE-SCIEX API-III (Thornhill, Ontario) triple quadrupole mass spectrometer with a pneumatically assisted electrospray ionozation source. Samples were flow injected in 50\% aqueous acetonitrile that contained $1 \%$ acetic acid for the positive ion mode. Collision of the protonated molecules with Ar gas at $60-\mathrm{eV}$ energy produced fragment ions which were mass analyzed in the third quadrapole. Raman spectra of HPLCpurified NDS, at neutral $\mathrm{pH}$, were kindly provided by Drs. Warner $\mathrm{L}$. Peticolas and Gerald A. Thomas of the University of Oregon, and phosphorous content was determined by a colorimetric assay as previously described (12).

\section{Results}

The HPLC assay shown in Fig. 1 yielded several absorbance peaks and NDS activity was contained in late fractions well removed from the fraction containing authentic adenosine. A tracing of column effluent absorbance at $254 \mathrm{~nm}$ is shown in Fig. 1 and the asterisk in this figure identifies the retention volume corresponding to authentic adenosine. To verify that the NDS activity so isolated from HL-60 cells corresponds to the NDS activity associated with supernates obtained from activated neutrophils, the fractionation patterns of NDS from these two sources were compared. DEAE HPLC elution profiles of the bioactive fraction were identical whether the source of NDS was from buffer conditioned with activated human peripheral blood neutrophils or from HL-60 lysates (Fig. 2). Preincubation of the crude NDS preparation with either adenosine deamidase or 5'-adenylic acid deamidase ablated the activity while incubation with adenosine 5 '-triphosphatase had no effect and $S$-adenosyl homocysteine hydrolase resulted in a

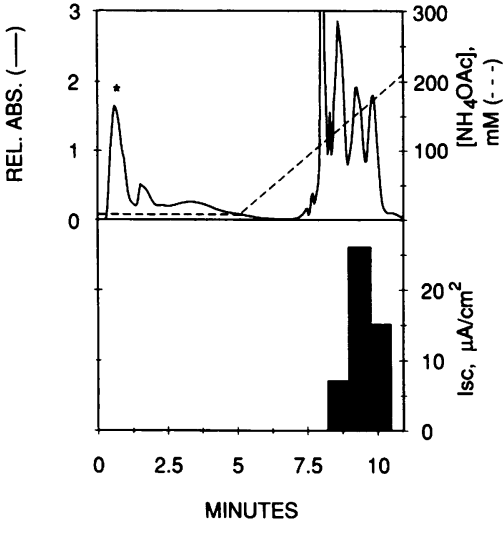

Figure 1. Chromatographic isolation of NDS bioactivity. Initial purification was accomplished by ultrafiltration and DEAE HPLC anion exchange chromatography as indicated in Methods. A tracing of HPLC column effluent absorbance at $254 \mathrm{~nm}$ is shown in the upper panel. Retention volume of authentic adenosine is represented by the asterisk. NDS activity, assessed by a bioassay which measured the $I_{s c}$ generated in response to apical exposure of T84 monolayers, for individual fractions (diluted 1:12) is shown in the lower panel. Note that no NDS activity was observed with fractions which should have contained adenosine (asterisk in upper panel).

slight increase in apparent NDS activity $(0.6 \pm 0.2,2.2 \pm 0.4$, $18.3 \pm 4.3$, and $26.2 \pm 7.5 \mu \mathrm{Amp} / \mathrm{cm}^{2}$, respectively, for crude NDS preparations treated with these enzymes, vs $18.5 \pm 4.2$ for crude NDS and 2.5 \pm 0.4 for buffer controls; all $n=4-5$ ). These and additional analyses yielded the following results: $(a)$ ultra-

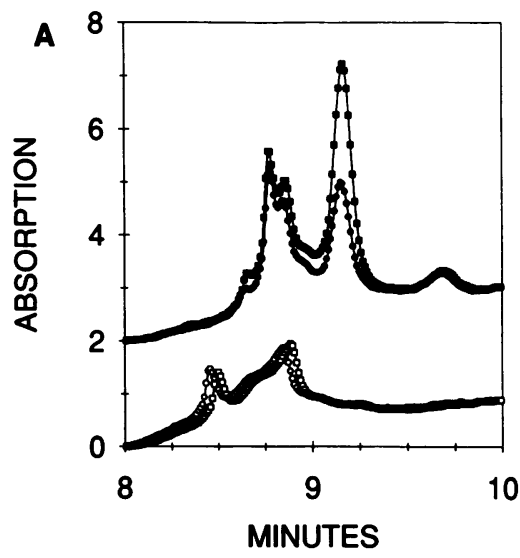

Figure 2. NDS derived from HL-60 cells displays chromatographic identity to that derived from neutrophil supernatants. $(A)$ HPLC elution profile of NDS bioactivity derived from activated primary neutrophils as described in the text. The NDS solution was diluted 10 -fold with either $1 \mathrm{mM}$ phosphate buffer $\mathrm{pH} 7.0$ (closed circles) or 100 nM 5'-AMP (closed squares) in the same

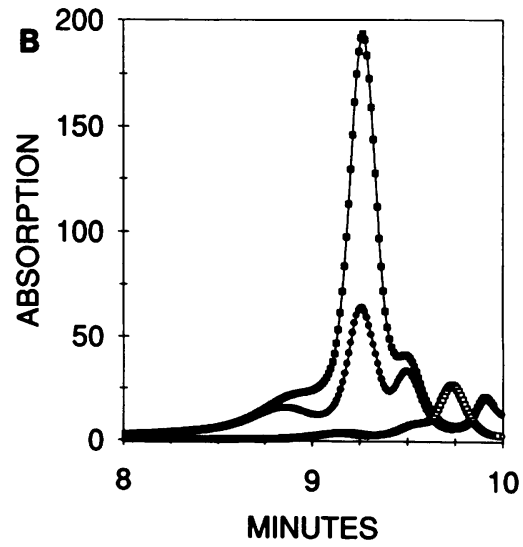
phosphate buffer. Incubation of diluted NDS solutions with 5'-nucleotidase $(1 \mathrm{U})$ is depicted by the open symbols. The estimated 5'-AMP concentration in the original primary neutrophil preparation is $1 \mu \mathrm{M}$. (B) HPLC elution profile of NDS bioactivity derived from HL-60 cells. The NDS was diluted 10 -fold with either $1 \mathrm{mM}$ phosphate buffer pH 7.0 (closed

circles) or $10 \mu \mathrm{M}$ 5'-AMP (closed squares) in the same phosphate buffer. Incubation of diluted NDS solutions with 5'-nucleotidase (1 $\mathrm{U}$ ) is depicted by the open symbols. The estimated 5'-AMP concentration in the original HL-60 preparation is $30-50 \mu \mathrm{M}$. 
violet absorption spectra demonstrated a shift in absorption maxima from 256.1 to $258.0 \mathrm{~nm}$ under acidic and basic conditions, respectively, consistent with the shift observed for authentic adenosine; $(b)$ nonresonance Raman spectra (at neutral $\mathrm{pH})$ demonstrated strong bands at 1,308, 1,337, and 1,378 $\mathrm{cm}^{-1}$ suggesting the presence of an adenine-like molecule; $(c)$ a parent compound of molecular mass $347 \mathrm{D}$ (nominal mass) was found by electrospray ionization mass spectrometry (13) and protonated $(\mathrm{m} / \mathrm{z} 348)$ and deprotonated $(\mathrm{m} / \mathrm{z} 346)$ molecules were detected in positive and negative ion modes, respectively (Fig. 3); (d) collision-induced dissociation of the protonated molecule produced an abundant fragment with $\mathrm{m} / \mathrm{z} 136$, strongly suggesting the adenine base, and the parent molecule as well as all minor product ions were identical to those observed in the fragment ion spectrum of a 5'-adenosine monophosphate standard (Fig. 3 ); ( $e$ ) phosphorous, determined by a colorimetric assay as previously described (12), was found to be present in roughly equimolar quantities with adenine (de-

\section{A}

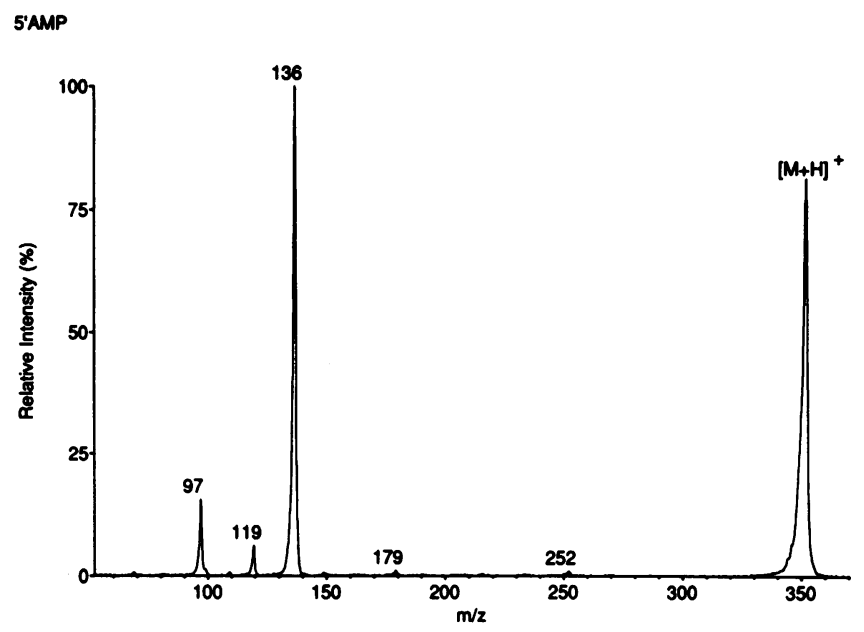

B

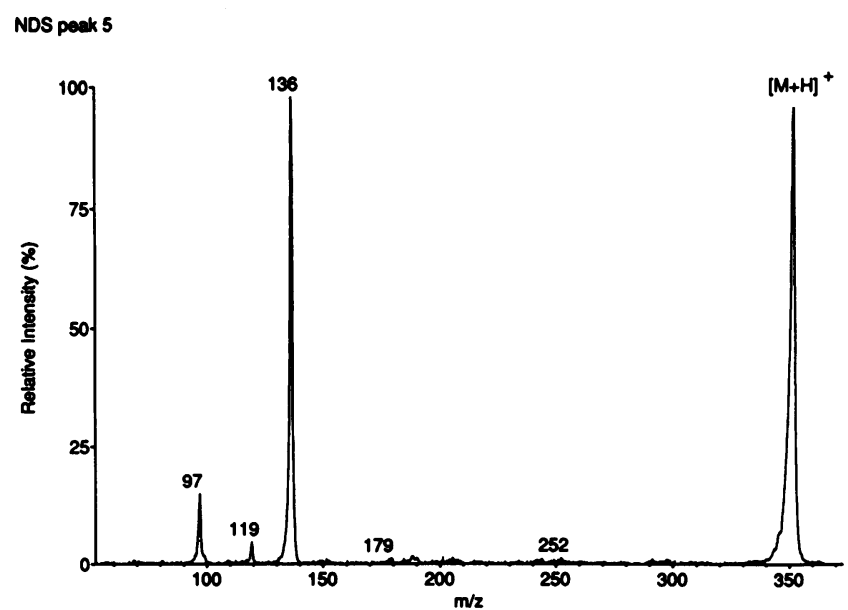

Figure 3. Collision-induced dissociation mass spectra of protonated 5'-AMP and purified NDS. $(A)$ 5'-AMP spectrum reveals the parent molecule $(\mathrm{m} / \mathrm{z} 348)$ and an abundant product ion at $\mathrm{m} / \mathrm{z} 136$ corresponding to the protonated adenine base. $(B)$ Purified NDS shares an identical spectrum to 5'-AMP. termined from ultraviolet absorption). Together these results suggest the identity of NDS to be a monophosphate form of adenosine. To resolve which monophosphate form(s) of adenosine represented NDS, fractionation patterns of purified monophosphates were examined. Injection of authentic adenosine 5'-monophosphate (5'-AMP), but not 3'- or 2'-AMP, onto the DEAE column resulted in an elution pattern identical to fractions demonstrating NDS activity (Fig. 2). Lastly, incubation of either neutrophil or HL-60-derived NDS with 5'-nucleotidase, ablated the DEAE HPLC absorbance peak in which the native bioactivity resided (Fig. 2, open symbols).

Given the above findings, 5'-AMP was used as a tentative identification of NDS. We next tested a series of molecules chemically similar to 5'-AMP for NDS activity (Table I). As shown, an intact adenosine moiety appeared to be required for full activity. Deoxy analogues of the reagents shown in Table I failed to have any activity as did uridine analogues or guanine (data not shown ). Addition of 5'-nucleotidase to either neutrophil or HL-60-derived NDS preparations ablated the HPLC peak in which the original bioactivity resided (Fig. 2). 5'-AMP showed activity in the T84 bioassay similar to that of NDS purified by the above procedure. Time course, dose response, and sideness studies revealed the striking similarity between 5'-AMP and purified NDS (Fig. 4). Interestingly, $I_{s c}$ responses to purified NDS and 5'-AMP were inhibited by the adenosine receptor antagonist 8-phenyltheophyline as effectively as adenosine (Fig. 5). These findings suggested the possibility of epithelial cell surface conversion of 5'-AMP to a subsequent effector (adenosine). Extracellular catabolism of 5'-AMP to adenosine

Table I. Effect of Structural Analogues to 5'-AMP in $I_{s c}$ Responses in $T_{84}$ Monolayers (9)

\begin{tabular}{lc}
\hline \multicolumn{1}{c}{ Analogue } & $\begin{array}{c}\text { Isc, } \mu \mathrm{A}-\mathrm{cm}^{-2} \\
(x \pm \mathrm{SEM} ; \boldsymbol{n}=4)\end{array}$ \\
\hline 5'-AMP & $42 \pm 8$ \\
3'-AMP & $41 \pm 6$ \\
2'-AMP & $18 \pm 4$ \\
2'-d3'AMP & $3 \pm<1$ \\
5'-AMS & $3 \pm<1$ \\
5'-dAMP & $4 \pm 1$ \\
5'-o-thio-AMP & $38 \pm 7$ \\
5'-ADP & $40 \pm 7$ \\
5'-dADP & $3 \pm 1$ \\
ATP & $33 \pm 3$ \\
5'-dATP & $3 \pm<1$ \\
ATP gamma-S & $3 \pm<1$ \\
3'5'-cdAMP & $2 \pm<1$ \\
3'5'-cAMP & $2 \pm<1$ \\
2'3'-cAMP & $23 \pm 4$ \\
2'-dAdenosine & $3 \pm<1$ \\
Adenosine & $45 \pm 7$ \\
3'-UMP & $2 \pm<1$ \\
5'-UMP & $3 \pm<1$ \\
5'-UTP & $3 \pm<1$ \\
Guanine & $4 \pm<1$ \\
Negative control (buffer) & $3 \pm<1$ \\
Purified NDS & $44 \pm 6$ \\
& \\
\hline
\end{tabular}

Peak (4-min) responses are reported for purine analogues. 

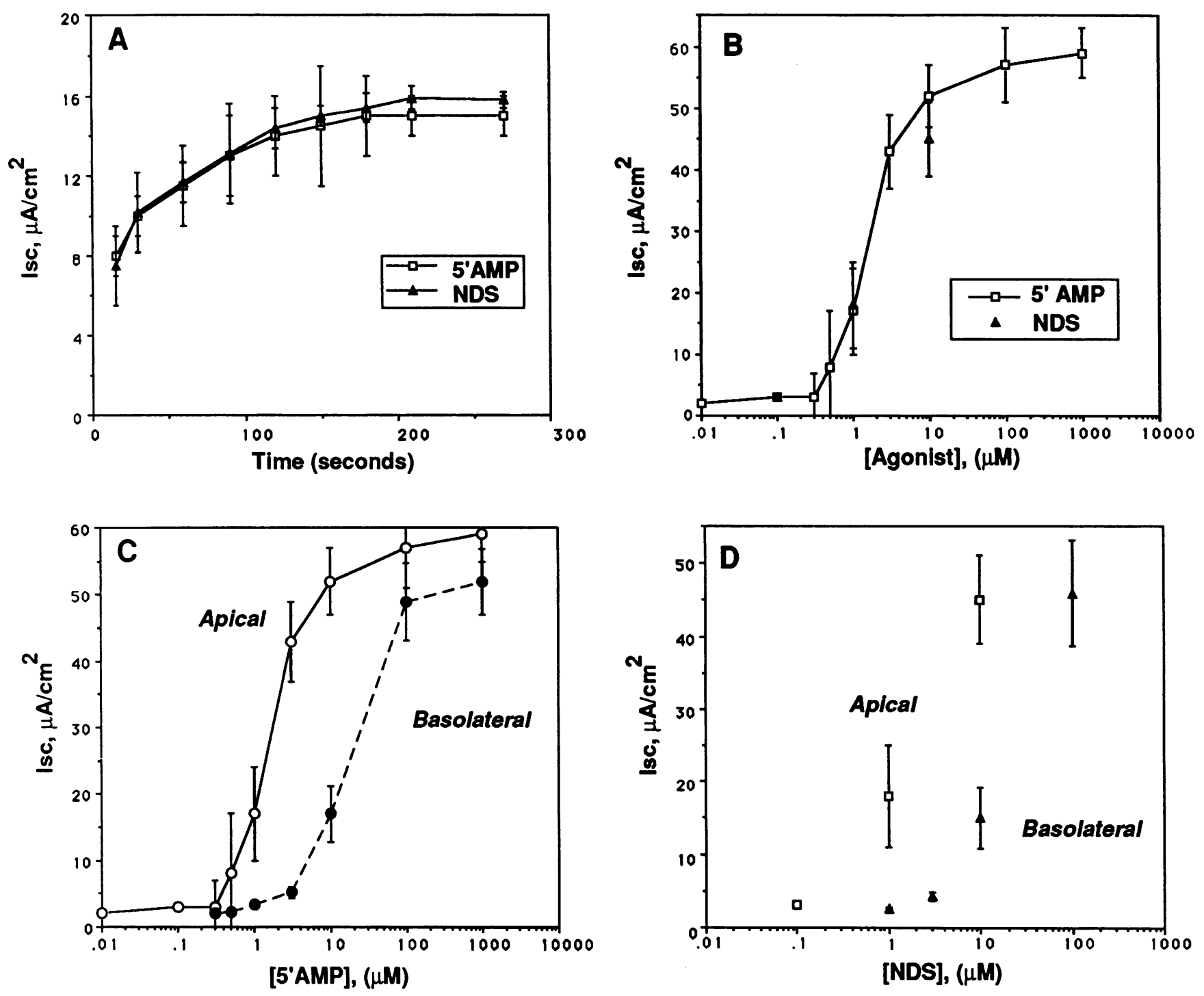

Figure 4. Characterization of T84 cell monolayer response to NDS and other agonists. NDS was purified as shown in Fig. 1 and reconstituted in HBSS. [NDS] was calculated from weight of purified material and molecular weight as determined by mass spectroscopy. Peak (4-8 min) $I_{s c}$ responses are shown. Agonists were applied to the apical side unless otherwise indicated. $(A, B)$ Time course ( $1 \mu \mathrm{M}$ agonist) and dose response to purified NDS compared to that of 5'-AMP. $(C, D)$ Sidedness of dose response to purified NDS compared to that of $5^{\prime}$-AMP $($ All points $n=4-7)$.

is known to be carried out by an ectoenzyme, ecto- 5 '-nucleotidase $(14,15)$. The inhibitor of 5 '-ecto-nucleotidase activity, $\alpha-\beta$ methylene-ADP, strongly inhibited the $I_{\text {sc }}$ response to $5^{\prime}$-AMP but not adenosine (Fig. 5 ).

It thus appears that the ability of 5 -AMP to elicit NDS activity at the epithelial surface of T84 cells occurs, at least in part, because of metabolic processing of 5'-AMP to adenosine which subsequently recognizes adenosine receptors. Interestingly, DEAE HPLC column fractions of HL-60 lysates or freshly isolated and activated neutrophil supernantants, collected at a retention time which correlated to authentic adenosine, showed no activity in the bioassay with T84 cell monolayers. Thus insufficient authentic adenosine was released by neutrophils to contribute to the secretory response under these conditions. The RP-HPLC method was used to examine the relative molar concentrations of adenosine and 5'-AMP in neutrophil supernatants. Control and spiking experiments with authentic adenosine and 5'-AMP suggested that the 5'-nucleotid- ase used in this assay ( see Methods) led to essentially complete conversion of 5'-AMP to adenosine, thus allowing for quantitation of components present in NDS preparations. Using this RP-HPLC assay it was found that, in supernatants from activated neutrophils, 5'-AMP was present in 10-100 $\times$ molar excess to authentic adenosine. DEAE experiments using spiking of NDS containing supernatants with 5'-AMP ( such as shown in Fig. 2) revealed that 5 -AMP concentrations in primary neutrophil supernatants were $\sim 1 \mu \mathrm{M}$ and those in HL-60 lysates were $30-50 \mu \mathrm{M}$. This correlated with dilutional bioassays which revealed $\sim 30 \times$ greater NDS bioactivity in ultrafiltrates of HL-60 lysates as compared with ultrafiltrates of primary neutrophil supernatants (7).

\section{Discussion}

Using intestinal epithelial bioassay systems it has been shown that many agents, known to be produced by neutrophils, are 

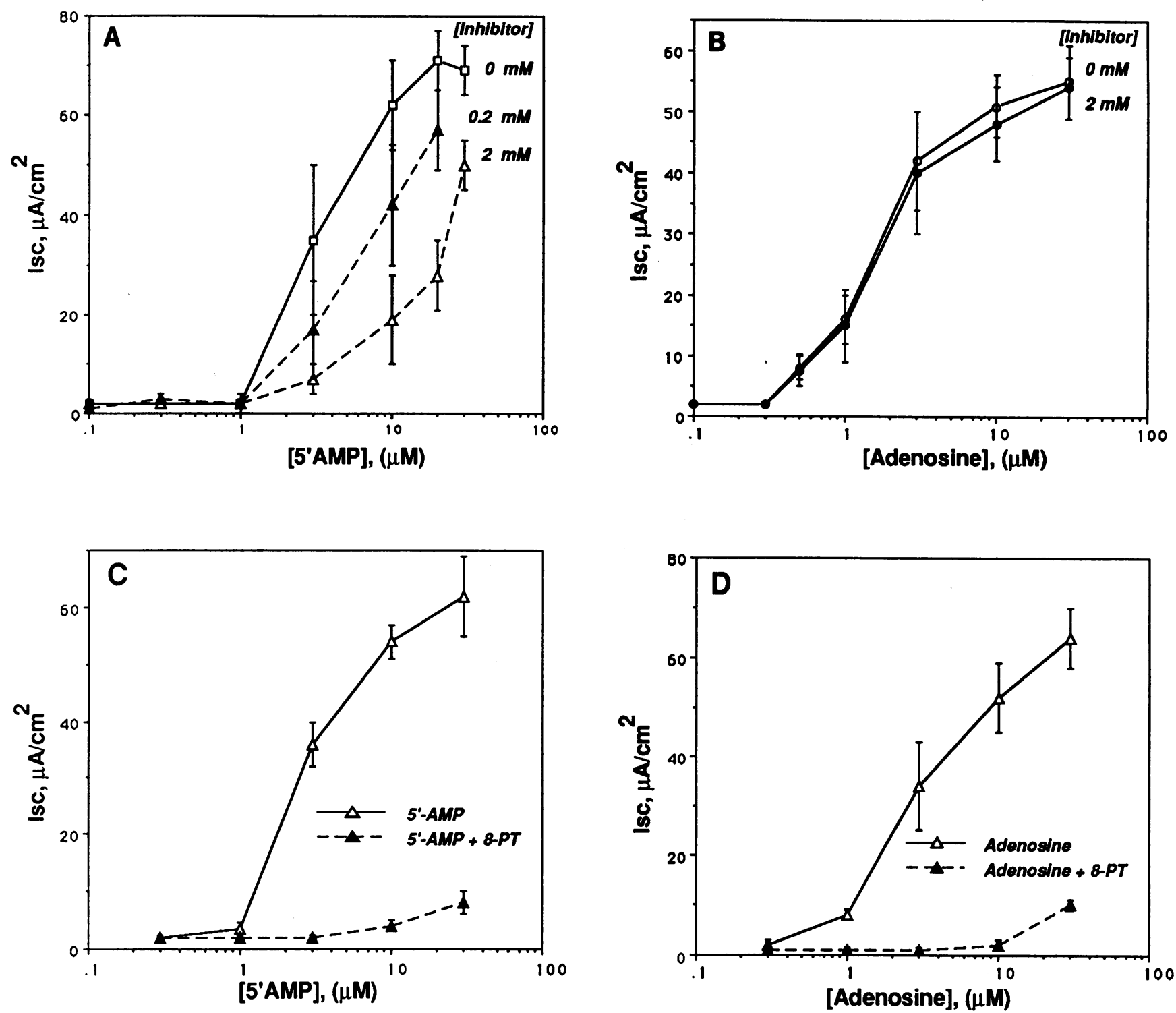

Figure 5. Characterization of relative contributions of epithelial apical membrane 5'-ectonucleotidase and adenosine receptors on the $\mathrm{I}_{\mathrm{sc}}$ response elicited by 5 '-AMP or authentic adenosine. $(A, B)$ Effect of 5 '-ectonucleotidase inhibitor, $\alpha-\beta$-methylene ADP, on $\mathrm{I}_{\mathrm{sc}}$ response elicited by 5 '-AMP (or NDS, data not shown) or authentic adenosine. $(C, D)$ Effect of adenosine receptor antagonist 8-phenyltheophylline $(10 \mu \mathrm{M})$ on $5^{\prime}$-AMP (or NDS, data not shown) or authentic adenosine (all points $n=4-7$ ).

capable, in pure form, of stimulating electrogenic $\mathrm{Cl}$ - secretion $(8-10)$. These current studies show that the NDS activity released from stimulated neutrophils which acts directly on intestinal epithelial cells is identical to that obtained from lysates of HL-60 cells. Moreover NDS bioactivity represents 5'-AMP. Studies using reconstituted HPLC fractions of NDS-conditioned buffer, measurement of 5'-AMP concentrations in these fractions, and analyses of 5'-AMP dose-response relationships for $\mathrm{Cl}$ secretion, indicate that the majority of secretagogue activity in neutrophil or HL-60-conditioned buffer represents 5'AMP. Studies of the relative molar concentration of adenosine and 5'-AMP in supernatants of activated neutrophils also indicate that the major adenine-containing moiety released by neutrophils is 5'-AMP, not authentic adenosine. Since we and others have previously shown that isolated neutrophils maintain a high degree (5) of viability, it is likely that 5'-AMP is released by neutrophils either in this or a precursor form such as ATP. It is presently unclear what process would eventuate in selective release of such compounds, although recent unpublished data indicate that specific membrane transport proteins may participate in the movement of ATP across biomembranes (13a).

Active intestinal inflammation is characterized by crypt abcesses in which numerous neutrophils, directly in contact with lumenal-activating factors $(3,5)$, have free access to the apical membrane of crypt epithelial cells. We have previously reported that NDS is primarily effective when applied to this apical domain $(5,6)$. Purification of NDS now shows it to be an effective secretagogue from both apical and basolateral domains although it is approximately an order of magnitude more effective apically. Since it appears from bioactivities and direct HPLC-based measurements that 5'-AMP is present in micromolar concentrations in neutrophil supernatants, since neutrophils induce an $I_{s c}$ when added apically but not basolat- 
erally to T84 monolayers, and since micromolar 5'-AMP is sufficient to stimulate secretion apically but not basolaterally, it is likely that this agonist may be most effective when presented apically as in a crypt abscess. We also present evidence that neutrophil-derived 5'-AMP is converted at the apical membrane to adenosine. By its activity and sensitivity to $\alpha-\beta$ methylene ADP, this apical 5'-AMPase activity appears to represent $\mathrm{CD} 73$, a cluster differentiation antigen now known to represent a glycosyl phosphatidylinositol-linked 5'-AMPase ectoenzyme (ecto-5'-nucleotidase) which has recently been cloned and sequenced (14). Like many polarized mammalian cells, T84 cells target glycosyl phosphatidylinositol-linked membrane proteins, such as alkaline phosphatase, to the apical membrane (Kaoutzani, P., C. Parkos, and J. L. Madara, manuscript in preparation). Thus the distribution of this enzyme, along with functionally defined adenosine receptors, to the apical membrane would be expected. Recently defined monoclonal antibodies which immunostain ecto-5'-nucleotidase have been used to show dense immunoreactivity on the apical membranes of crypt (and surface) epithelial cells in the human intestine (15). Thus the presence of this enzyme on the apical membrane of T84 cells recapitulates the natural condition found in crypt epithelia.

Based on the evidence presented here, the following hypothesis of NDS-elicited Cl-secretion, outlined in Fig. 6, emerges: neutrophils in crypt abcesses release 5'-AMP which is

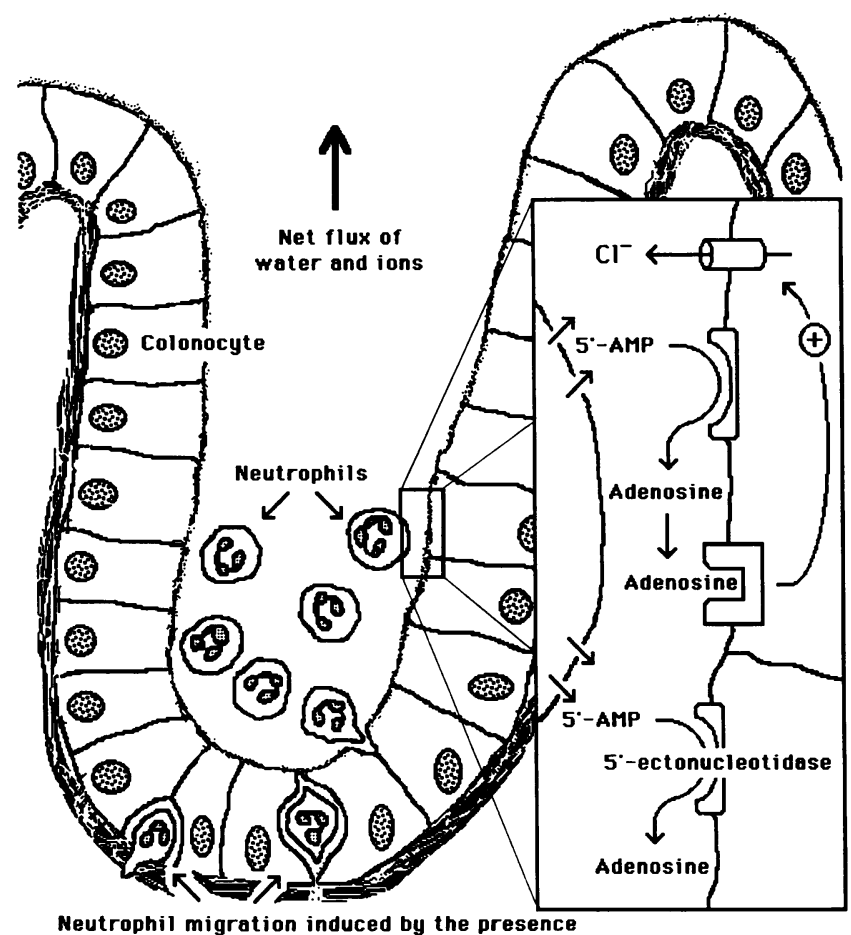

Figure 6. Putative mechanism by which NDS induces isotonic volume flushing of the crypt lumen. Translocation of PMN into the luminal compartment is signaled to the epithelial apical membrane in paracrine fashion by 5 -AMP (NDS). At the epithelial cell surface the 5'-AMP is efficiently translated into an adenosine signal by 5 '-ectoectonucleotidase. The resulting epithelial secretory response permits the crypt lumen to flush itself of the chemotactic challenge. Such events may permit sequential coupling of these two defenses (neutrophil transmigration and surface volume flush). converted to adenosine by the epithelial ecto-5'-nucleotidase residing on the apical membrane. Locally released adenosine could then serve as an effector for secretion by interacting with its receptor on this same domain. The resulting volume flush of the crypt lumen would serve to clear the crypt of the threat that initially stimulated neutrophil transepithelial migration. Thus 5'-AMP may function as a novel "paracrine" mediator that contributes to fluid secretion in active intestinal inflammation. If this hypothesis proves correct, better understanding of the regulation of the interactive components of this model could identify new strategies for the treatment of diarrhea associated with intestinal inflammation.

\section{Acknowledgments}

This work was supported by National Institutes of Health grants DK35932 and DK-33506 (project 4). Dr. Parkos is recipient of a Career Development Award from the Crohn's and Colitis Foundation of America. Dr. Colgan is supported by National Institutes of Health training grant HL-07627.

\section{References}

1. Halm, D. R., and R. A. Frizzell. 1990. Intestinal chloride secretion. In Textbook of Secretory Diarrhea. E. Lebentahl, editor. Raven Press, Ltd., New York. 47-58

2. Welsh, M. J., P. L. Smith, M. Fromm, and R. A. Frizzell. 1982. Crypts are the site of intestinal fluid and electrolyte secretion. Science (Wash. DC). 218:1219-1221.

3. Kumar, N. B., T. T. Nostrant, and H. D. Appleman. 1982. The histologic spectrum of acute self-limited colitis. Am. J. Surg. Pathol. 6:523-529.

4. Dharmsathaphorn, K., J. A. McRoberts, K. G. Mandel, L. D. Tisdale, and H. Masui. 1984. A human colonic tumor cell line that maintains vectoral transport. Am. J. Physiol. 246:G204-G208.

5. Nash, S., C. Parkos, A. Nusrat, C. Delp, and J. L. Madara. 1991. In vitro model of intestinal crypt abscess. J. Clin. Invest. 87:1474-1477.

6. Madara, J. L., C. A. Parkos, S. Nash, J. B. Matthews, C. Delp, and W. Lencer. 1992 . $\mathrm{Cl}$ secretion in a model intestinal epithelium induced by a neutrophil-derived secretagogue. J. Clin. Invest. 89:1938-1944.

7. Colgan, S. P., J. B. Matthews, C. A. Parkos, C. Delp, C. S. Awtrey, and J. L. Madara. 1992. The myeloid leukemia cell line HL-60 produces a factor that induces chloride secretion in cultured epithelial cells. J. Leukocyte Biol. 52:183187.

8. Karayalcin, S. S., C. W. Sturbaum, J. T. Wachsman, J. H. Cha, and D. W. Powell. 1990. Hydrogen peroxide stimulates rat colonic prostaglandin production and alters electrolyte transport. J. Clin. Invest. 86:60-68.

9. Tamai, H., J. F. Kachur, D. A. Baron, M. D. Grisham, and T. S. Gaginella 1990. Monochloramine, a neutrophil-derived oxidant stimulates rat colonic secretion. J. Pharmacol. Exp. Ther. 257:884-894.

10. Tamai, H., T. S. Gaginella, J. F. Kachar, M. W. Musch, and E. B. Chang. 1992. Ca-mediated stimulation of $\mathrm{Cl}$ secretion by reactive oxygen metabolites in human colonic T84 cells. J. Clin. Invest. 89:301-307.

11. Parkos, C. A., C. Delp, M. A. Arnaout, and J. L. Madara. 1991. Neutrophil migration across a cultured intestinal epithelium: dependence on a CD1 lb/ CD-18-mediated event and enhanced efficiency in the physiological direction. $J$. Clin. Invest. 88:1605-1612.

12. Mrsny, R. J., J. J. Volwerk, and O. H. Griffith. 1986. A simplified procedure for lipid phosphorus analysis shows that digestion rates vary with phospholipid structure. Chem. Phys. Lipids. 39:185-191.

13. Fenn, J. B., M. Mann, C. K. Meng, S. F. Wong, and C. M. Whitehouse. 1990. Mass spectroscopy. Mass Spectroscopy 9:37-70.

13a. Abraham, E. H., A. G. Prat, L. Gerweck, T. Seneveratne, R. J. Arceci, R. Kramer, G. Guidotti, and H. F. Cantiello. 1993. The multidrug resistance ( $m d r l$ ) gene product functions as an ATP channel. Proc. Natl. Acad. Sci. USA. 90:312316.

14. Misumi, Y., O. Shigenori, S. Hirose, and Y. Ikehara. 1990. Primary structure of rat liver 5'-nucleotidase deduced from the cDNA. J. Biol. Chem 265:2178-2183.

15. Thompson, L. F., J. M. Ruedi, A. Glass, G. Moldenhauser, P. Moller, M. G. Low, M. R. Klemens, M. Massaia, and A. H. Lucas. 1990. Production and characterization of monoclonal antibodies to the glycosyl phophatidylinositolanchored lymphocyte differentiation antigen ecto-5'-nucleotidase (CD73). Tissue Antigens. 35:9-19. 